Review

\title{
Responses to the Standard for Exchange of Nonclinical Data (SEND) in non-US countries
}

\author{
Takayuki Anzai $^{1 *}$, Masamichi Kaminishi ${ }^{1}$, Keizo Sato $^{1}$, Laura Kaufman ${ }^{2}$, Hijiri Iwata ${ }^{3}$, \\ and Dai Nakae ${ }^{* * *}$ \\ ${ }^{1}$ Showa University School of Medicine, 1-5-8 Hatanodai, Shinagawa, Tokyo 142-0064, Japan \\ ${ }^{2}$ PDS Life Sciences, 100 Valley Road, Suite 204, Mt. Arlington, NJ 07856, U.S.A. \\ ${ }^{3}$ LunaPath LLC, Aoihigashi 3-5-1, Naka-ku, Hamamatsu-shi, Shizuoka 433-8114, Japan \\ ${ }^{4}$ Department of Nutritional Science and Food Safety, Faculty of Applied Biosciences, Tokyo University of Agriculture, 1-1-1 Sakura- \\ ga-Oka, Setagaya, Tokyo 156-8502, Japan
}

\begin{abstract}
The Standard for the Exchange of Nonclinical Data (SEND), adopted by the US FDA, is part of a set of regulations and guidances requiring the submission of standardized electronic study data for nonclinical and clinical data submissions. SEND is the nonclinical implementation of SDTM (Study Data Tabulation Model), the standard electronic format for clinical regulatory submissions to FDA. SEND, SDTM, and the associated Controlled Terminology have been developed by CDISC (Clinical Data Interchange Standards Consortium). In order to successfully implement SEND, interdisciplinary contributions between sponsors and CROs, need a model for task allocation. This is being undertaken by the Pharmaceutical Users Software Exchange (PhUSE). Because SEND is currently the preferred submission format of the US FDA only and will become required by it starting in December 2016, only American academic societies and companies are actively involved. An exception to this is the INHAND initiative, which leads the way in standardizing terminology for toxicological pathology. On the other hand, international globalization of other clinical and nonclinical practices is not feasible because there are substantial differences between the US and non-US countries in CRO involvement in drug development. Thus, non-US countries must consider and develop approaches to SEND that meet their needs. This paper summarizes the activities of the major organizations involved in SEND development and implementation, discusses the effective use of SEND, and details a compliance scheme (research material of the Showa University School of Medicine) illustrating how pharmaceutical companies can complete a large amount of work up to an FDA application with the effective utilization of CROs and solution providers. (DOI: 10.1293/ tox.2015-0007; J Toxicol Pathol 2015; 28: 57-64)
\end{abstract}

Key words : SEND, CDISC, FDA, INHAND, controlled terminology

\section{Introduction}

Globally, countries are actively computerizing medical product registration. In Japan, the Pharmaceuticals and Medical Devices Agency (PMDA), with the support of the Japan Pharmaceutical Manufacturers Association (JPMA), has started computerizing clinical study data and performed a pilot project ${ }^{1}$ for computerizing application screens. It has also started to recruit personnel in anticipation of the future introduction of SEND and the SDTM. Obviously, the US FDA is staying ahead of other countries in clinical study

Received: 26 January 2015, Accepted: 28 January 2015

Published online in J-STAGE: 1 April 2015

*Corresponding author: T Anzai (e-mail: takayuki.anzai@me.com)

**Co-corresponding author: D Nakae

(e-mail: agalennde.dai@nifty.com)

(C)2015 The Japanese Society of Toxicologic Pathology

This is an open-access article distributed under the terms of the Creative Commons Attribution Non-Commercial No Derivatives (by-ncnd) License $<$ http://creativecommons.org/licenses/by-nc-nd/3.0/> . and preclinical study data computerization and standardization. As a result, patients have greatly benefited from the speedier approval of new drugs, and the US pharmaceutical industry has been able to maintain its advantage over rivals in other countries. According to research conducted by a Yale University study group ${ }^{2}$, among three administrative agencies, FDA, European Medicines Agency (EMA), and Health Canada, the period (median value) from application to completion of the first examination (2001-2010) was shortest for FDA. FDA is trying to further shorten the review period by standardizing the electronic formats for clinical and preclinical study submissions using the SDTM and SEND, developing electronic tools to analyze and visualize these submissions, and building data warehouses to rapidly query data across drugs, companies, and clinical and nonclinical disciplines. This infrastructure is already in place and being used by FDA. 
Table 1. Step-by-step Approaches by INHAND (GESC), FDA, CDISC, and EVS

\begin{tabular}{ll}
\hline Phase I & -Classify and define the terms used in SEND \\
$2012-2014$ & -Position INHAND terminology as SEND terminology and perform a proper mapping \\
\hline & $\begin{array}{l}\text {-Appoint experts on different areas to the committee to hear their opinions and update requests regarding } \\
\text { INHAND terminology, and coordinate the process of updating terms }\end{array}$ \\
$\begin{array}{l}\text { Phase II } \\
\text { in progress }\end{array}$ & $\begin{array}{l}\text {-Develop a framework of future activities in partnership with various academic journals including goRENI } \\
\text { (global open Registry Nomenclature Information system). Also, work with the FDA and other organiza- } \\
\text { tions that are working on the development of SEND terminology }\end{array}$ \\
\hline Phase III & $\begin{array}{l}\text {-Develop the terminology of non-rodent species. Do that not by organ but species-by-species } \\
\text {-Set up expert panels for each of the following: dogs, monkeys, rabbits, and miniature pigs. Each panel } \\
\text { member compiles the terminology in a manuscript }\end{array}$ \\
\hline
\end{tabular}

\section{The Purpose of SEND and the Involvement of Related Organizations}

SEND is an application of the Clinical Data Interchange Standards Consortium (CDISC) Study Tabulation Model (SDTM) ${ }^{3}$ to nonclinical data submissions. SDTM is an electronic standard used when patient data listings for clinical studies are submitted to regulatory authorities. The purpose of SEND is to reduce the new drug application examination time and improve nonclinical data review and analysis by facilitating the creation of sophisticated analysis and visualization tools and data warehouses through data standardization. Data warehouses allow FDA (and sponsors who also create their own data warehouses) rapid access to large amounts of data. Is it possible to shorten the FDA review period by simply standardizing study data using SEND and its associated controlled terminology? The answer is no. However, standardized electronic study data facilitate the development and use of data analysis tools and data warehouses, which will result in efficient, rapid, and improved data review. As part of the standardized electronic formats, FDA requests the use of controlled terminology (CT). CDISC and academic societies including INHAND are working on development of this terminology, while international organizations like PhUSE are playing an important role in establishing the operational aspects of SEND.

\section{FDA and CDISC}

CDISC, a nonprofit standards development organization with representatives from pharmaceutical and biotech companies, CROs, vendors, consultants, and FDA, is responsible for developing the FDA-mandated electronic standards. According to FDA's position statement on study data standards for regulatory submissions, "FDA does not foresee the replacement of CDISC standards for study data and will not implement new approaches without public input on the cost and utility of those approaches" ${ }^{\text {,4 }}$. In December 2012, FDA announced a 5-year Information Technology Plan (draft $)^{5}$, revealing the outline of the promotion program for a series of electronic applications and computerized screenings. Most important, FDA issued the final guidance on electronic standards for SEND and the SDTM ${ }^{6}$ on December 17, 2014. This guidance will become binding on
December 17, 2016, for applicable NDAs, BLAs, and ANDAs and on December 17, 2017, for applicable INDs, The guidance discusses FDA's Data Standards Catalog, which provides a listing of supported and/or required exchange, study data, and controlled terminology standards along with important dates, such as when support or requirements begin and end for particular standards and versions. The Study Data Technical Conformance Guide ${ }^{7}$ includes points to consider regarding Study Data Reviewer's Guides, Study Data Standardization Plans, controlled terminologies, and other important elements of standardized electronic submissions. SEND Implementation Guide (SENDIG) 3.0 pertains to single- and repeated-dose toxicology studies and carcinogenicity studies. SENDIG 3.1 will include cardiovascular and respiratory safety pharmacology studies ${ }^{8}$, in addition to the studies covered by SEND 3.0. It should be noted that SEND will eventually be required for almost all nonclinical studies.

\section{INHAND}

INHAND is an international organization composed of toxicological pathology societies of the US, UK, Europe, and Japan that cooperatively work towards the standardization of terminology 9 . The importance of the INHAND terminology is recognized by CDISC, and INHAND's Global Editorial Steering Committee (GESC) is playing a leading role in the development of controlled terminology for SEND. A new CT version that will include INHAND terms for nonneoplastic lesions is expected to be released at about the same time as the SENDIG 3.1 release. As Table 1 shows, INHAND is currently working on a step-by-step basis ${ }^{10}$ in cooperation with FDA, CDISC, and Enterprise Vocabulary Services (EVS) of the National Cancer Institute.

Meanwhile, it should be noted that pathology is naturally a descriptive science, which always involves shades of gray in the expression of morphological changes. In SEND, all findings as reported by pathologists are required even if they do not use controlled terminology. The findings are then mapped to controlled terminology in separate columns within the SEND macroscopic and microscopic findings domains. All comments by pathologists are also captured in SEND in the using the pathologist's terminology.

Additionally, the terminologies provided by INHAND do not cover all the terminologies used in SEND. An enor- 
Table 2. Needs for SEND

\begin{tabular}{lll}
\hline When & From whom & Why \\
\hline Application for FDA (IND, NDA) & FDA & Requirements of regulatory authorities \\
\hline $\begin{array}{l}\text { Cut time to market } \\
\begin{array}{l}\text { Data trading in a joint development or } \\
\text { licensing-in/licensing-out }\end{array}\end{array}$ & Tll stakeholders & $\begin{array}{l}\text { Increased profit for pharmaceutical companies, faster } \\
\text { delivery of new drugs to patients }\end{array}$ \\
\hline $\begin{array}{l}\text { Preparation for SEND regulations of } \\
\text { many countries around the world }\end{array}$ & Authorities of each country & Future requirements \\
\hline Establishment of data warehouse & Sponsor companies & Bioinformatics, rapid access to data \\
\hline
\end{tabular}

mous amount of SEND terminology, including terminology for reproduction and teratology studies, will be added in the future.

\section{PhUSE and Interorganizational SEND (iSEND)}

PhUSE is an international nonprofit organization made up of mainly experts on data management, biostatistics, and electronic clinical data who are working for US and European pharmaceutical and information technology companies. It also includes representatives from FDA actively involved in SEND, the SDTM, and CDISC. PhUSE is working primarily to promote "best practices" with respect to SEND and SDTM implementation. One of the PhUSE SEND Working Groups is Interorganizational SEND (iSEND) ${ }^{11}$. This group is studying the allocation of roles between related organizations (sponsors and CROs), and scenarios that will help pharmaceutical companies cooperate efficiently with study institutions or related organizations to respond to SEND. One of the biggest challenges for SEND implementation stems from the use of multiple CROs or providers for a single toxicology study. For example, in-life and postmortem evaluation may be conducted at one $\mathrm{CRO}$, but bioanalytical and toxicokinetics evaluations may be performed in-house or at another CRO. The iSEND group is addressing the integration of SEND datasets from multiple organizations into one consistent SEND dataset with consistent metadata and controlled terminology versions across domains. Such integration is necessary for SEND compliance.

\section{Relationship of SEND and the SDTM with the eCTD}

The eCTD or electronic Common Technical Document is a separate electronic standard for pharmaceutical submissions developed by the International Conference on Harmonization of Technical Requirements for Registration of Pharmaceuticals for Human Use (ICH). ICH is comprised of regulatory agencies across Europe, Japan, and the US. Adherence to eCTD formats will become an FDA requirement twenty-four months following issuance of FDA's final guidance on this subject ${ }^{12}$. When SEND datasets are submitted as part of an eCTD submission, they will be part of Module 4 (Nonclinical Study Reports), as described in the
Study Data Technical Conformance Guide?

\section{SEND for the EU}

At the present time, the EU does not have formal plans to adopt SEND or SDTM standardized electronic formats. However, many pharmaceutical companies in the EU have already started preparing for the SDTM and SEND. In fact, the number of PhUSE members from the EU is now larger than the number of US members.

\section{Needs for SEND}

Compared with other regulations, SEND is unique because pharmaceutical companies can use it for various purposes, which can be broadly divided into five categories (Table 2).

Besides applying for FDA approval, which is one obvious purpose, there have recently been cases where new product benefits could be obtained with a shorter examination time, which is an advantage provided by standardized electronic formats (SEND and the SDTM). That is, increased economic and medical benefits can occur due to a faster time to market, as well as when a business partner's request is met through data traded in joint development or licensing-in/licensing-out of drugs. In a co-development project, the handling of data will be easier for both companies if data of both sides are prepared in the format of SEND (or SDTM) dataset. Also in the case of licensing-out, the partner will be more likely to request SEND (or SDTM) dataset in the future. In addition, since the governments of Japan and European countries may eventually introduce standardized electronic formats (SEND and SDTM), another purpose will be to prepare a response to the SEND regulations of many countries across the world. This preparation includes obtaining the knowledge and skill related to internal use of SEND. In a different context, several pharmaceutical companies have started to establish data warehouses using legacy data that has been converted to SEND and to utilize those data for bioinformatics and new drug development in the future. 


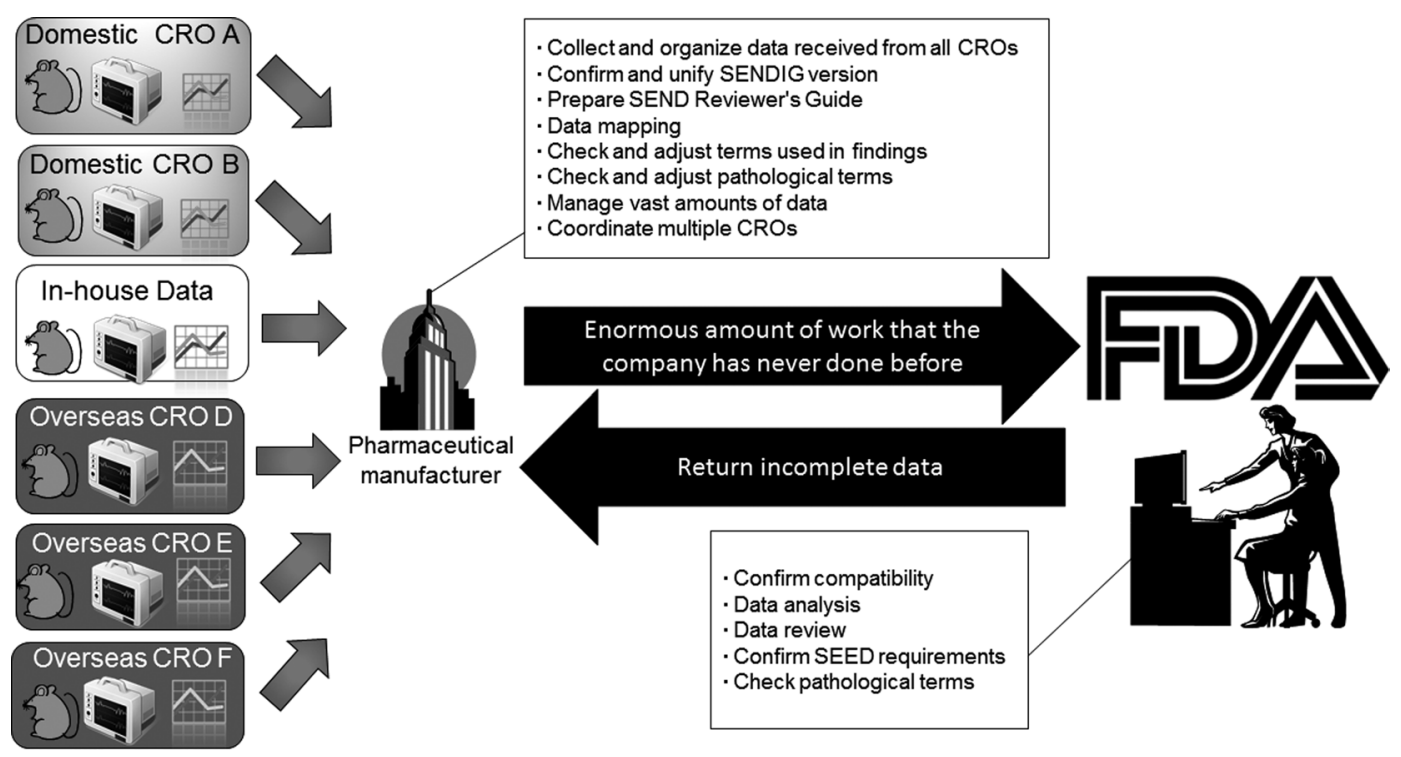

Fig.1. Current situation of SEND in non-US countries. Non-US pharmaceutical companies need to conform the data created in multiple countries and facilities to the SEND requirements.

\section{Challenges in Responding to SEND in Japan and Other Non-US Countries and Ways to Manage Them}

\section{Challenge 1: Differences in study entrustment}

Generally, most pharmaceutical companies that are based or have headquarter functions in the US conduct safety tests of drugs at their own research facilities and CROs. Accordingly, in iSEND, the assumption is that roles for SEND are shared among US-based CROs. This can be considered a region-based model in which US pharmaceutical companies apply to the US authority using US CROs. Meanwhile, in Japan, the situation varies greatly. For example, as Fig. 1 shows, most Japanese pharmaceutical companies often use more than one Japan-based CRO as well as CROs based in Europe and the US for the development of one product. This means they need to conform the data created in multiple countries and facilities to the SEND requirements. In these cases, not all the commissioned CROs can necessarily use SEND or use it in a manner that meets the client's needs. Another aspect that needs to be taken into account is the analyses and tests contracted out by CROs to subcontractors.

\section{Challenge 2: Reliability of SEND datasets}

Pharmaceutical companies take full responsibility for the reliability of SEND datasets. CROs bear Good Laboratory Practice (GLP) responsibilities for the reliability of raw data or original electronic data, but currently not for the reliability of the SEND data converted from the original data. Obviously, CROs assume no responsibility, in terms of both laws and GLP, for the preparation of applications or the compatibility of electronic data with FDA's screening tool. Therefore, SEND datasets are not recommended to be sub- mitted by CROs for application without verification. This point is sufficiently discussed in the iSEND.

\section{Challenge 3: Flexibility of $C R O s$}

If a CRO is using a widely used converter for SEND, the program will be updated as needed to deal with the addition of CTs and the requirements of FDA, which are expected to increase in the future. However, if the CRO is using a self-developed program, the sponsor always needs to verify that the program is accurate and updated. In any case, since CROs bear GLP responsibilities for the original data, the important thing is how they can submit the original data in an electronic format to meet the sponsor's needs. Sponsor pharmaceutical companies should confirm how flexible commissioned CROs can be in the submission of before-conversion original data in the proper electronic format, rather than requesting them to submit the dataset in the SEND format.

\section{Challenge 4: Accountability to FDA}

The scenario being studied by iSEND is predicated based on the idea that pharmaceutical companies are equipped with expertise in SEND and converters, and capable of properly evaluating a SEND dataset. In fact, however, the iSEND scenario is not currently applicable to all pharmaceutical companies or all CROs. CDISC has announced the specialized organizations related to SEND in the CDISC Registered Solution Provider (RSP) Chart ${ }^{13}$. Pharmaceutical companies can receive assistance from RSPs as needed to fulfill their accountability to the authorities. Using RSPs is helpful particularly for pharmaceutical companies in nonUS countries. There is concern, however, that the number of RSPs is limited, while demand for RSPs is expected to increase further. 


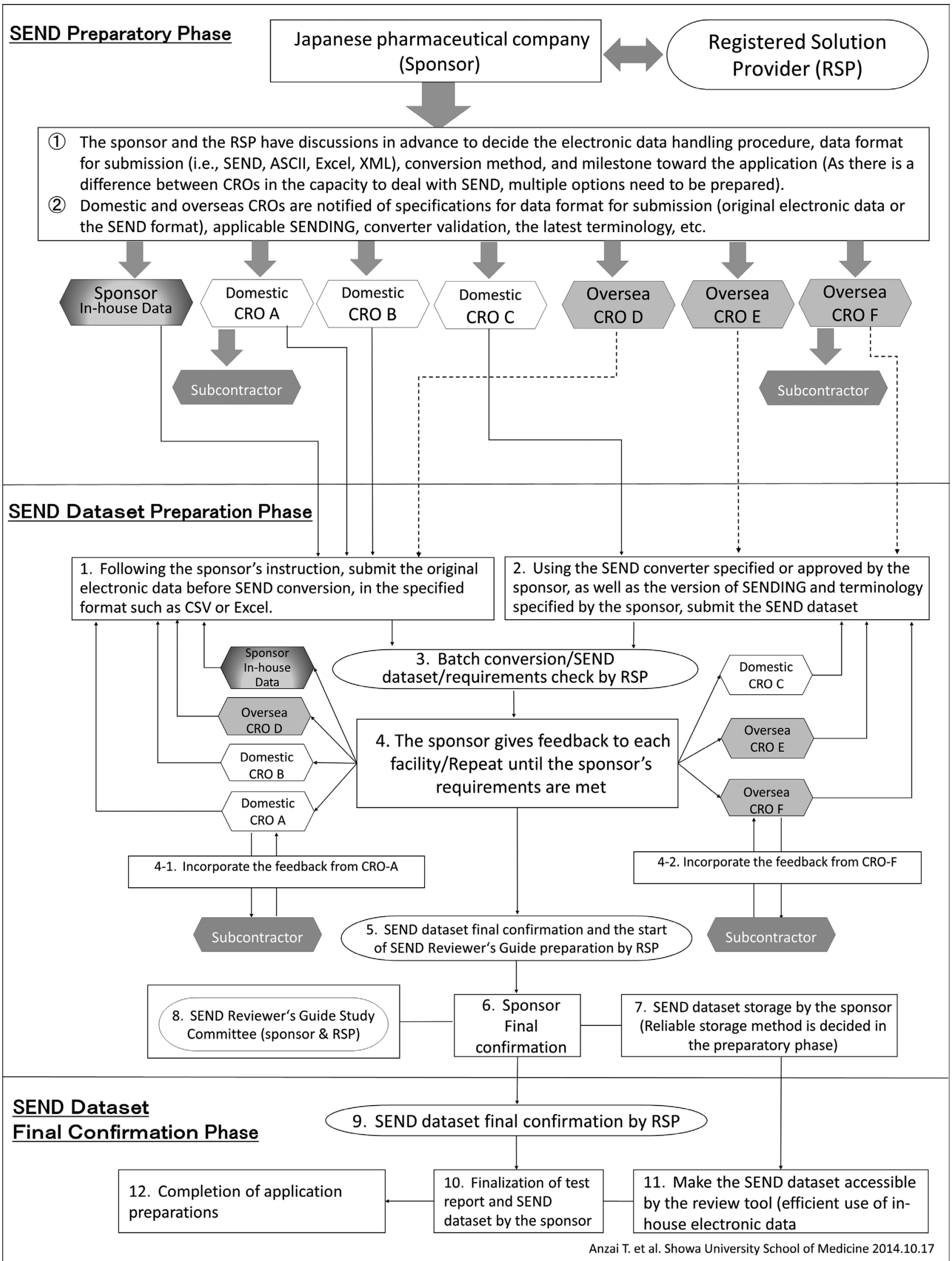

Fig. 2. SEND compliance scheme. How non-US pharmaceutical companies can overcome challenges by using RSPs. 


\section{Efficient Ways for Japanese Pharmaceutical Companies to Respond to SEND}

Given the current situation in Japan, it is difficult to imagine a scenario in which both pharmaceutical companies and CROs have sufficient knowledge of SEND and a capacity to manage it. In addition, as discussed earlier, a region-based scenario is not applicable to Japanese pharmaceutical companies.

Fig. 2 (SEND compliance scheme in Japan and other non-US countries) illustrates how Japanese or other non-US countries' pharmaceutical companies can overcome challenges by using RSPs. One of this scheme's characteristics is that the phases from SEND dataset preparation to final confirmation are described based on the use of CROs with different nationalities and capacities to use SEND.

\section{SEND Preparatory Phase}

In the SEND preparatory phase in Fig. 2 (top), the sponsor pharmaceutical company consults with an RSP to summarize the proper requirements while taking into account each CRO's capacity to comply with SEND (Fig. 2, SEND Preparatory Phase (1) and (2)). In this phase, if there are any tests or studies outsourced by a CRO to a subcontractor, the necessary arrangements need to be made to ensure that the CRO properly informs the subcontractor of the sponsor's requirements.

\section{SEND Dataset Preparation Phase}

The SEND dataset preparation phase in Fig. 2 (middle) starts with classification of the data, which were received from CROs into two forms according to their capacities (Steps 1 and 2). Here, the sponsor's own data are also included.

If a CRO follows the sponsor's instruction and submits before-conversion electronic data (Step 1), proceed to the step (Step 3) in which the RSP confirms the data received and converts them. On the other hand, for a dataset submitted by a CRO that can submit a SEND dataset in the manner approved by the sponsor, the RSP confirms whether such data meet the requirements of SEND and the sponsor. It is, of course, necessary to sufficiently confirm beforehand whether the CRO can meet these specifications.

In Fig. 2, Steps 2 and 3, Steps 1, 3 and 4, or Steps 2-4 are repeated until the sponsor's requirements are met. Similar processes are repeated between the $\mathrm{CRO}$ and its subcontractors until the requirements are met.

After completing the final confirmation, the RSP starts to prepare a SEND Study Data Reviewer's Guide (Step 5). At this point, the sponsor sets up a SEND Study Data Reviewer's Guide Study Committee (Step 8), which consists of the sponsor and the experts from organizations such as the RSP, and also makes arrangements to store the SEND dataset before it is finalized (Step 7). The sponsor submits to the RSP the SEND Study Data Reviewer's Guide approved by the committee. The SEND Study Data Reviewer's Guide explains to FDA examiners how the SEND data were converted and how the dataset is structured for each test, and it must be attached to the SEND dataset for each study.

\section{SEND Dataset Final Confirmation Phase}

The SEND dataset final confirmation phase in Fig. 2 (bottom) is an important phase in which the sponsor verifies the compatibility of the SEND dataset with the FDA's screening system. The RSP submits the SEND dataset before finalization to FDA for a trail submission, in which the FDA's validation tools are used to verify that no compliance errors are found in the dataset that will interfere with processing and analyzing the electronic data (Step 9). If an error is found here, the data must be reviewed by going back to the SEND dataset preparation phase in Fig. 2. In this case, the SEND dataset is not finalized for storage. If the SEND dataset passes the FDA trail submission, the study report is immediately finalized, and the SEND dataset is finalized for storage and FDA submission.

\section{Storage and Browsing of the SEND Dataset}

The dataset before finalization, which was stored in the SEND dataset final confirmation phase in Fig. 2 (Step 7), or the finalized dataset needs to always be accessible by the sponsor or RSP with the SEND dataset review tool (Step 11).

\section{Points to be Noted When Pharmaceutical Com- panies Work with CROs}

At the moment, CROs assume no responsibility for SEND in terms of both laws and GLP. In reality, however, sponsor pharmaceutical companies may request CROs to convert data into SEND data. This causes CROs to introduce conversion systems or to outsource conversion to external organizations including RSPs. However, it is not realistic for CROs, which currently assume no GLP responsibility, to be involved directly in tasks such as data conversion, confirmation of SEND dataset compatibility, preparation of a SEND Study Reviewer's Guide, and coordination with CROs based at home and abroad. If a Japanese CRO, among others, is to manage these tasks, it will need to utilize an internationally accepted SEND converter, and establish a department consisting of experts on SEND as well as coordinators to work with Japanese and overseas CROs. Obviously, this specialized department must be proficient in the terminology and English.

On the other hand, if a pharmaceutical company is to use SEND without depending on CROs, the necessary organizational response will be as described above.

If a CRO states that it can offer assistance from preparation of a SEND dataset to submission of an FDA application, pharmaceutical companies should confirm in advance these points: 1) if the CRO is equipped with an internationally accepted SEND converter capable of reflecting updated 
information from organizations including FDA, CDISC, INHAND, and PhUSE, as well as related know-how; 2) if the CRO has a department to support SEND, consisting of bilingual experts on SEND; 3) if the CRO has a track record of preparing SEND Study Reviewer's Guides; and 4) if the CRO can bear the contractual liability until the FDA trial application.

The worst CROs to work with are those that work until the conversion into a SEND dataset is completed, but take no responsibility beyond that point. In this case, the pharmaceutical company will have to bear a greater burden because sufficient verification and analysis are needed for converter validation, terminology updates, data integrity after conversion, and applicable SENDIG versions. Thus, it is preferable to request that the $\mathrm{CRO}$ submit the original data in the specified electronic format, as described in Fig. 2 (Step 1).

\section{Risk of Application Rejection}

The largest risk associated with SEND is that of a refusal to file (RFP) from FDA. FDA clearly states that it will return the SEND dataset to the applicant if FDA's validation system identifies errors. This means applications may be returned before they are reviewed. Therefore, application materials, including the SEND dataset and accompanying Reviser's Guide, need to be complete. If the application is returned, the launch of a product will be delayed, causing a significant loss.

Upon receipt of a submission, FDA tries to complete the validation process within a specified period of time. If FDA receives a SEND dataset that contains validation errors, it may return the dataset to the sponsor for correction. Some validation errors can prevent analysis by FDA. For this reason, the FDA is strict about the integrity of datasets and does not hesitate to return incomplete ones.

\section{Conclusion}

Even if an application with a SEND dataset is returned for reexamination, CROs and vendors will not be held responsible. It is obviously the applicant pharmaceutical company that takes on the responsibility and associated risks. The largest challenge related to SEND is how pharmaceutical companies can avoid these risks in an adequate manner. Although we think the compliance scheme (Fig. 2) explained in this paper is a very practical way to deal with SEND in Japan or other non-US countries, it is only one of many ways. With increased response capacities of pharmaceutical companies in the future, more efficient ways should be devised. The biggest advantage of SEND is its potential to decrease the examination time, which means, a faster time to market. Although it will not be officially introduced until December 2016, FDA is already accepting submissions with SEND and SDTM datasets, and it has expressed that these standardized formats are preferred., Many pharmaceutical companies are using this system, and this is probably because SEND and SDTM are viewed not as a regulatory requirement but as a business strategy tool. Pharmaceutical companies now need strategic thinking to avoid the risks involved in using SEND as well as to enjoy its benefits.

Declaration of Conflicting Interests: The authors have no conflicts of interest to be disclosed in relation to this paper.

\section{References}

1. Yamori T. Center for Product Evaluation of Pharmaceuticals and Medical Devices Agency. Request for Electronic Clinical Study Data for Pilot Project. PMDA/CPE Notification No. 0902001 September 2, 2013., from PMDS web site: http://www.pmda.go.jp/operations/shonin/info/iyaku/ jisedai/file/tsuuchi_e.pdf.

2. Downing NS, Aminawung JA, Shah ND, Braunstein JB, Krumholz HM, and Ross JS. Regulatory review of novel therapeutics - comparison of three regulatory agencies. N Engl J Med. 366: 2284-2293. 2012. [Medline] [CrossRef]

3. CDISC SDTM TEAM Clinical Data Interchange Standards Consortium (CDISC). The Study Data Tabulation Model (SDTM) Implementation Guide version 3.2. CDISC SDTM TEAM. 2013, from CDISC web site: http://www.cdisc.org/ sdtm.

4. U.S. Food and Drug Administration Study Data Standards for Regulatory Submissions Position Statement. September 13, 2013, from US FDA web site: http://www.fda.gov/forindustry/datastandards/studydatastandards/ucm368613.htm.

5. U.S. Food and Drug Administration Center for Drug Evaluation and Research. Office of Reguraoly Affaires. GDUFA Information Technology/Informatiics Plan (Draft) FY 2013 - FY 2017. September 2014, from FDA web site: http:// www.fda.gov/downloads/ForIndustry/UserFees/GenericDrugUserFees/UCM416856.pdf.

6. U.S. Food and Drug Administration Center for Drug Evaluation and Research. Center for Biologics Evaluation and Research. Providing Regulatory Submissions in Electronic Format -Standardized Study Data.Guidance for Industry. December 2014, from FDA web site: http://www.fda.gov/ RegulatoryInformation/Guidances/ucm126959.htm.

7. US Department of Health and Human Services U.S. Food and Drug Administration. Center for Drug Evaluation and Research. Center for Biologics Evaluation and Research. STUDY DATA TECHNICAL CONFORMANCE GUIDE: Technical Specification Document. Guidance for Industry Providing Regulatory Submissions in Electronic Format Standardized Study Data. December 2014, from FDA web site: http://www.fda.gov/downloads/Drugs/Guidances/ UCM292334.pdf.

8. CDISC SEND TEAM, Clinical Data Interchange Standards Consortium Standard for Exchange of Nonclinical Implementation Guide (IG) Version 3.1 for Public Review as of Dec. 4, 2014, from CDISC web site: http://www.fda.gov/ downloads/ForIndustry/DataStandards/StudyDataStandards/UCM384744.pdf.

9. Dixon D, Alison R, Bach U, Colman K, Foley GL, Harleman JH, Haworth R, Herbert R, Heuser A, Long G, Mirsky M, Regan K, Van Esch E, Westwood FR, Vidal J, and Yoshida M. Nonproliferative and proliferative lesions of the rat and mouse female reproductive system. J Toxicol Pathol. 27(Suppl): 1S-107S. 2014. [Medline] [CrossRef] 
10. International Harmonization of Nomenclature and Diagnostic Criteria for Lesions in Rats and Mice INHAND. Collaboration with the FDA on SEND: Standard for the Exchange of Nonclinical Data. 2012, from Society of Toxcologic Pathology web site: https://www.toxpath.org/STP inhand- $84 \times 42 v 2 \% 20$ pdf.pdf.

11. Interorganizational SEND. (iSEND). Pharmaceutical Users Software Exchange (PhUSE). Scenarios for Generating SEND Datasets from Nonclinical Safety Studies Conducted Across Multiple Organizations. Interorganizational SEND. White Paper. 2013, from PhUSE web site: http://www. phusewiki.org/wiki/index.php?title=Interorganizational_ SEND_-_Scenarios_White_Paper.
12. U.S. Department of Health and Human Services U.S. Food and Drug Administration. Center for Drug Evaluation and Research. Center for Biologics Evaluation and Research. Guidance for Industry. Providing Regulatory Submissions in Electronic Format. Certain Human Pharmaceutical Product Applications and Related Submissions Using the eCTD Specifications. Draft - Revision 3 - July, 2014, from FDA web site: http://www.fda.gov/downloads/drugs/guidancecomplianceregulatoryinformation/guidances/ucm333969. pdf.

13. Clinical Data Interchange Standards Consortium (CDISC) CDISC Solution Provider Chart. 2014, from CDISC website: http://www.cdisc.org/rsp-chart. 